\title{
Erratum to: Selective serotonin reuptake inhibitors versus placebo in patients with major depressive disorder. A systematic review with meta-analysis and Trial Sequential Analysis
}

\author{
Janus Christian Jakobsen ${ }^{1,2^{*}}$, Kiran Kumar Katakam', Anne Schou', Signe Gade Hellmuth', Sandra Elkjær Stallknecht',

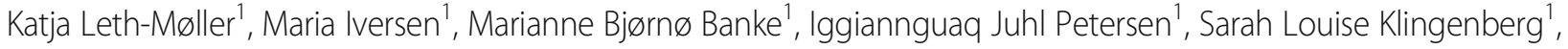 \\ Jesper Krogh ${ }^{3}$, Sebastian Elgaard Ebert ${ }^{1}$, Anne Timm ${ }^{1}$, Jane Lindschou ${ }^{1}$ and Christian Gluud ${ }^{1}$
}

\section{Erratum}

After the publication of this paper the authors noticed a few errors in the text. There are outlines below:

1. Results section, under Hamilton depression rating scale (HDRS): In the sentence 'Nineteen trials reported only mean HDRS change scores or presented a graph showing the mean change HDRS scores, but did not report the SD' Eighteen should replace Nineteen

2. Results section, under Hamilton depression rating scale (HDRS):In the sentence 'Random-effects metaanalysis of the results of all $\mathbf{9 2}$ trials showed that SSRIs versus placebo significantly reduced the HDRS score (mean difference -2.25 points; $95 \%$ CI -2.69 to $-1.83 ; \mathrm{P}<0.00001$ )' 91 should replace $\mathbf{9 2}$

3. Results section, under Other subgroup analyses: In the sentence 'Meta-analysis of the results of the $\mathbf{2 6}$ trials with a mean baseline HDRS score $>23$ points showed a mean difference of -2.69 HDRS points; $95 \%$ CI -3.59 to $-1.78 ; \mathrm{P}<0.00001$. 25 should replace $\mathbf{2 6}$

4. Table 2 [Summary of serious adverse events in the included trials] 'Nemroff et al., 2005' should read 'Nemeroff et al., 2007' and in the same line 'Fluxetine' should read 'Fluoxetine'

\section{Author details}

${ }^{1}$ The Copenhagen Trial Unit, Centre for Clinical Intervention Research, Department 7812 Rigshospitalet, Copenhagen University Hospital, Blegdamsvej 9, Rigshospitalet, 2100 Copenhagen, DK, Denmark. ${ }^{2}$ Department of Cardiology, Holbæk Hospital, Holbæk, Denmark. ${ }^{3}$ Mental Health Centre Copenhagen, Faculty of Health Sciences, University of Copenhagen, Copenhagen, Denmark.

Received: 23 March 2017 Accepted: 12 April 2017 Published online: 03 May 2017

\section{Reference}

1. Jakobsen JC, Katakam KK, Schou A, Hellmuth SG, Stallknecht SE, Leth-Møller K, Iversen M, Banke MB, Petersen IJ, Klingenberg SL, Krogh J, Ebert SE, Timm A, Lindschou J, Gluud C. Selective serotonin reuptake inhibitors versus placebo in patients with major depressive disorder, A systematic review with meta-analysis and Trial Sequential Analysis. BMC Psychiatry. 2017;17:58. doi:10.1186/s12888-016-1173-2.

\footnotetext{
*Correspondence: jcj@ctu.dk

${ }^{1}$ The Copenhagen Trial Unit, Centre for Clinical Intervention Research, Department 7812 Rigshospitalet, Copenhagen University Hospital, Blegdamsvej 9, Rigshospitalet, 2100 Copenhagen, DK, Denmark

${ }^{2}$ Department of Cardiology, Holbæk Hospital, Holbæk, Denmark
} 